Two Di st i nct Cyl i nder Arrangement s i n Monodomai ns of a Lyot ropi c Li qui d Cryst al I i ne Hexagonal II Phase : Monodomai ns wi th Strai ght Cyl i nders and Ri nged Cyl i nder s i n Capi I I ar i es

\begin{tabular}{|l|l|}
\hline 著者 & Oka Toshi hi ko, Oht a Nobor u \\
\hline $\begin{array}{l}\text { j our nal or } \\
\text { publ i cat i on ti l l e }\end{array}$ & Langmi $r$ \\
\hline vol une & 32 \\
\hline nunber & 30 \\
\hline page r ange & $7613-7620$ \\
\hline year & $2016-07-11$ \\
\hline 出版者 & Aner i can Chemi cal Soci et y \\
\hline 権利 & Copyr i ght ( C) 2016 Amer i can Chem cal Soci et y \\
\hline 注記 & ACS Aut hor Choi ce \\
\hline URL & ht t p: //hdl . handl e. net /10297/10351 \\
\hline
\end{tabular}




\title{
Two Distinct Cylinder Arrangements in Monodomains of a Lyotropic Liquid Crystalline Hexagonal II Phase: Monodomains with Straight Cylinders and Ringed Cylinders in Capillaries
}

\author{
Toshihiko Oka*t, ${ }^{*}$ and Noboru Ohta ${ }^{\S}$ \\ ${ }^{\dagger}$ Department of Physics, Faculty of Science and ${ }^{\ddagger}$ Nanomaterials Research Division, Research Institute of Electronics, Shizuoka \\ University, Shizuoka 422-8529, Japan \\ ${ }^{\S}$ SPring-8/JASRI, 1-1-1 Kouto, Sayo-cho, Sayo-gun, Hyogo 679-5198, Japan
}

\section{Supporting Information}

ABSTRACT: We report a method to produce two different monodomains of an inverse hexagonal II $\left(\mathrm{H}_{\mathrm{II}}\right)$ phase in capillaries. Capillaries filled with glyceryl monooleyl ether (GME) in an inverted micellar phase were soaked in water. After a week, a monodomain of the $\mathrm{H}_{\text {II }}$ phase with straight cylinders was observed in a capillary with a diameter of $1.0 \mathrm{~mm}$. The axis of the straight cylinders was almost parallel to the capillary axis, and the cylinders were slightly undulated. The lattice constant of the $\mathrm{H}_{\mathrm{II}}$ phase was $5.85 \mathrm{~nm}$, which indicated the monodomain was fully hydrated. Another monodomain with ringed cylinders was observed in a 0.2 $\mathrm{mm}$ diameter capillary. The ringed cylinders aligned to the round capillary wall, where one of the $\langle 10\rangle$ directions in the hexagonal lattice always faced the wall. The lattice constant was $4.89 \mathrm{~nm}$, from which the estimated water content of the monodomain was almost the lowest reported for the $\mathrm{H}_{\mathrm{II}}$ phase. The monodomain with ringed cylinders is stabilized by the capillary wall and the low water content. This method to produce specific monodomains is expected to be of benefit for basic and applied research on the $\mathrm{H}_{\mathrm{II}}$ phase.

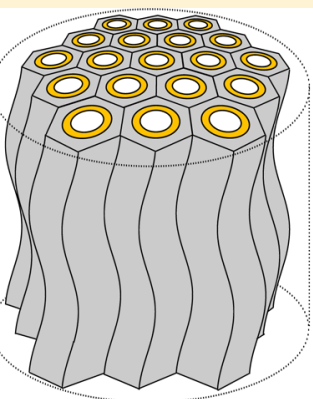

straight cylinders

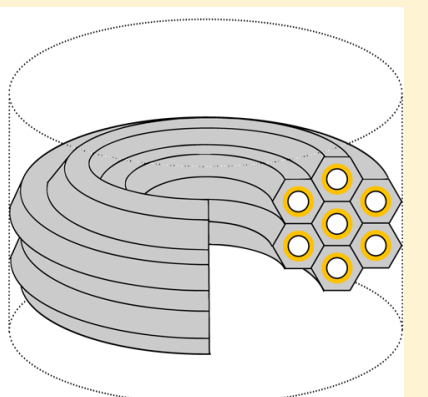

ringed cylinders

\section{INTRODUCTION}

The hexagonal phase is frequently observed in lyotropic liquid crystals. ${ }^{1}$ Amphiphile molecules in the hexagonal phase are selfassembled into cylindrical micelles and stack to form a twodimensional (2D) hexagonal lattice (Figure 1). The arrangement of amphiphile molecules in cylindrical micelles is type I (oil-in-water) in the normal hexagonal $\mathrm{I}\left(\mathrm{H}_{\mathrm{I}}\right)$ phase, while it is type II (water-in-oil) in the inverse hexagonal II $\left(\mathrm{H}_{\mathrm{II}}\right)$ phase. ${ }^{2,3}$

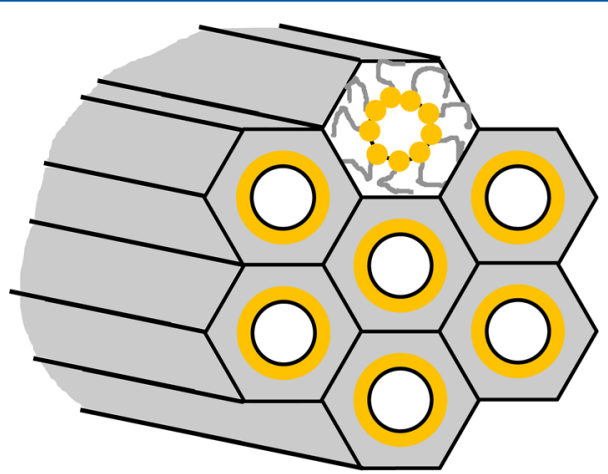

Figure 1. Cross section of the $\mathrm{H}_{\text {II }}$ phase structure. Inverse cylinders of amphiphiles are stacked to form a $2 \mathrm{D}$ hexagonal lattice. The cylindrical axis is perpendicular to the hexagonal lattice plane.

\section{.}

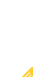

Both hexagonal phases have highly ordered nanostructures where hydrophilic and hydrophobic regions are aligned at the nanoscale. Applications of hexagonal phases have been developed to use these nanostructures, the most successful of which is the use of the $\mathrm{H}_{I}$ phase as templates to produce mesoporous structures ${ }^{4,5}$ and nanowire arrays. ${ }^{6}$ In addition, nanoparticles of the $\mathrm{H}_{\text {II }}$ phase, referred to as hexosomes, are used in the field of drug delivery researches. ${ }^{7,8}$

Samples containing randomly oriented domains have been used in much of the research on the hexagonal phase. However, some techniques have been reported for the preparation of monodomain samples of the $\mathrm{H}_{\mathrm{I}}$ phase, such as alignment in the gap between two glass plates ${ }^{9,10}$ and by shear force. ${ }^{11,12}$ In addition, there are techniques reported for alignment of the $\mathrm{H}_{\mathrm{II}}$ phase, such as oriented sample preparation on flat silicon plates ${ }^{13}$ and on the inner surface of a capillary. ${ }^{14}$ However, to the best of our knowledge, no methods have been reported for the preparation of a monodomain of the $\mathrm{H}_{\mathrm{II}}$ phase. Monodomain samples generally have different properties than their polydomain equivalents. For example, there is no perturbation generated at the domain boundaries. Rancon

Received: March 13, 2016

Revised: June 3, 2016

Published: July 11, 2016 
and Charvolin reported the epitaxial relationships during phase transformations using a monodomain sample of phases including the $\mathrm{H}_{\mathrm{I}}$ phase. ${ }^{9}$ Templating with a monodomain can result in different properties from that with polydomains. Therefore, we have focused on the preparation of a monodomain of the $\mathrm{H}_{\mathrm{II}}$ phase.

We have previously developed a method to produce a single crystal region of the inverse bicontinuous cubic phase in a capillary. ${ }^{13}$ With the method, slow dilution of an organic solvent from an open edge of the capillary caused a slow phase transition from the $L_{3}$ phase to the inverse cubic phase. The $\mathrm{L}_{3}$ phase does not have a stable structure and can be treated as a viscous liquid during sample preparation. Here, we present a method for the preparation of monodomains of the $\mathrm{H}_{\text {II }}$ phase in capillaries using the phase transition from the inverse micellar $\left(\mathrm{L}_{2}\right)$ phase to the $\mathrm{H}_{\text {II }}$ phase.

\section{MATERIALS AND METHODS}

Glyceryl monooleyl ether (GME; NIKOL Selachyl alcohol, Nikko Chemicals Co. Ltd., Tokyo, Japan) was used without further purification. GME forms the $\mathrm{L}_{2}$ phase at $25{ }^{\circ} \mathrm{C},{ }^{16}$ and can thus be treated as a viscous liquid. GME was loaded into quartz glass capillaries (Mark-tube, Hilgenberg GmbH, Germany) with diameters of 0.2 or $1.0 \mathrm{~mm}$. The $0.2 \mathrm{~mm}$ diameter capillary has variations in the diameter of approximately $30 \%$. The length of GME in the capillary was ca. 30 $\mathrm{mm}$. One end of the sample was sealed with a tube sealing compound (Chaseal, Chase Scientific Glass, TN), while the other end was soaked in water at $25{ }^{\circ} \mathrm{C}$. After approximately 1 week, the $\mathrm{H}_{\text {II }}$ phase was formed at the open side of the capillary. Some of the GME near the open end was pushed away from the capillary because the volume of GME was expanded by swelling with water. The $\mathrm{H}_{\text {II }}$ phase region was observed with a polarized-light microscope (BX-53P, Olympus, Tokyo, Japan), or a stereomicroscope (SZX-7, Olympus) with two polarizers (a polarizer and an analyzer). A capillary sample was placed between a slide glass and cover glass, and the gap between was filled with water.

$\mathrm{X}$-ray diffraction measurements with the $1.0 \mathrm{~mm}$ diameter capillary were conducted at a standard small-angle X-ray scattering beamline, BL40B2, at SPring-8 (Hyogo, Japan). The X-ray beam size was ca. H $0.4 \mathrm{~mm} \times \mathrm{V} 0.3 \mathrm{~mm}$ at the sample position. The capillary sample was mounted on a goniometer head with a rotating stage, as described in previous works. ${ }^{15,17,18}$ A 2D pixel array detector, (PILATUS-100K, Dectris, Baden, Switzerland) was used to obtain X-ray diffraction images. The distance between the sample and the detector was 668.5 $\mathrm{mm}$, and the X-ray wavelength was 0.1000 or $0.0826 \mathrm{~nm}$. Measurements were performed at room temperature $\left(26^{\circ} \mathrm{C}\right)$. X-ray diffraction was measured using the rotating crystal method, similar to that in previous studies. ${ }^{15,17,18}$ The sample was rotated continuously from 0 to $360^{\circ}$, and X-ray images with $1 \mathrm{~s}$ exposure and $2^{\circ}$ steps were measured sequentially. The X-ray diffraction images were obtained using ALBULA Viewer software (Dectris, Baden, Switzerland). The intensity distribution in reciprocal space was calculated from the sequential X-ray diffraction data and produced with Image ${ }^{19}$ and Volume Viewer plugins.

The X-ray microbeam diffraction measurements with the $0.2 \mathrm{~mm}$ diameter capillary were conducted at a high-flux beamline, BL40XU, at SPring-8. The beamline is not equipped with a monochromator, and $\mathrm{X}$-rays from a helical undulator are focused with horizontal and vertical mirrors. ${ }^{20}$ Higher-order X-rays are eliminated with the mirrors. As a result, the energy bandwidth of the first-order X-ray was approximately 1.6 to $5.2 \%$ (fwhm). The capillary sample was mounted in the same manner as that in the BL40B2 beamline experiment. A 2D X-ray detector was constructed using a scientific CMOS camera (C114022CU, Hamamatsu Photonics, Hamamatsu, Japan) coupled with an Xray image intensifier (V7739PMOD, Hamamatsu Photonics). The distance between the sample and detector was $725.1 \mathrm{~mm}$. The X-ray wavelength was $0.0826 \mathrm{~nm}$ and the energy bandwidth of the X-rays was approximately $2 \%$. Measurements were performed at room temperature $\left(26^{\circ} \mathrm{C}\right)$. A $5 \mu \mathrm{m}$ diameter pinhole was used to produce an X-ray microbeam, ${ }^{21}$ with which the capillary sample was scanned along the direction perpendicular to the capillary axis. Diffraction images were obtained using ImageJ. ${ }^{19}$

\section{RESULTS}

Capillary samples filled with GME were soaked in water for 3 weeks. The GME/water system forms the $\mathrm{L}_{2}$ phase at room temperature with a water content of up to $12 \%$, while it transforms into the $\mathrm{H}_{\text {II }}$ phase if the water content becomes larger than $13 \%{ }^{22}$ The transformation to the $\mathrm{H}_{\text {II }}$ phase also causes an increase in the volume of the sample, so that some of the GME is pushed away from the capillary The $L_{2}$ phase is optically isotropic, while the $\mathrm{H}_{\mathrm{II}}$ phase has anisotropic along the $c$-axis (cylindrical axis). The birefringence of the $\mathrm{H}_{\text {II }}$ phase present in the capillary could be easily recognized with a polarized light microscope. Thus, a bright region is the $\mathrm{H}_{\mathrm{II}}$ phase and a dark region is the $\mathrm{L}_{2}$ phase when directions of polarizer and analyzer plates of a polarized microscope are crossed. But, if the $c$-axis of the $\mathrm{H}_{\mathrm{II}}$ phase in a region is aligned, the region is dark when the aligned $c$-axis is parallel to the polarized light direction from polarizer or analyzer plates (extinction position). ${ }^{23}$ And the aligned region is the brightest when the angle between the aligned $c$-axis and the polarized light direction is $45^{\circ}$ (diagonal position). Figure 2 shows the $\mathrm{L}_{2}-\mathrm{H}_{\mathrm{II}}$ interface region in a $1.0 \mathrm{~mm}$ diameter capillary observed with a polarized light microscope. In Figure 2a, a clear interface was observed in the sample between the $L_{2}$ phase (a right region in the capillary) and the $\mathrm{H}_{\mathrm{II}}$ phase (a region from center to left) due to the difference in the refraction indexes of both phases when polarizer and analyzer directions were parallel. In the $\mathrm{H}_{\text {II }}$ phase region, a stripe pattern is observed perpendicular to the capillary axis. Figure $2 \mathrm{~b}$ shows the same region between crossed polarizer and analyzer plates, and the capillary axis was parallel to the polarized light from the polarizer. The capillary sample was almost dark, but with weak rainbow-colored stripes in the $\mathrm{H}_{\text {II }}$ region. The observation shown in Figure $2 \mathrm{c}$ was almost the same as in Figure $2 \mathrm{~b}$ but with the capillary rotated $45^{\circ}$. The $\mathrm{H}_{\mathrm{II}}$ region of the capillary was the brightest in Figure $2 c$. The color of the region was almost white, but various colors were observed at the edge near the $\mathrm{L}_{2}-\mathrm{H}_{\mathrm{II}}$ interface where the thickness of the $\mathrm{H}_{\mathrm{II}}$ phase gradually decreased. The angle of the capillary in Figure $2 \mathrm{c}$ was considered to be the diagonal position, while that in Figure $2 \mathrm{~b}$ was the extinction position. The results indicate that the $c$-axis of the $\mathrm{H}_{\text {II }}$ phase in the capillary is aligned along or perpendicular to the capillary axis. In Figure $2 c$, the various color stripes at the edge of the $\mathrm{H}_{\text {II }}$ phase are almost the same as the interference color chart (see a textbook about a polarized light microscope ${ }^{23}$ ). The same region was then observed with a first-order retardation plate. Slow axis orientation of the retardation plate was parallel to the capillary axis. In Figure $2 \mathrm{~d}$, a black stripe appeared at the $\mathrm{L}_{2}$ phase edge and various color stripes shifted toward the whitecolored $\mathrm{H}_{\text {II }}$ phase region. The optical sign of birefringence along the capillary axis of the sample was determined as negative. The length of the aligned $\mathrm{H}_{\mathrm{II}}$ region in Figure 2 was approximately $8 \mathrm{~mm}$ (Figure $\mathrm{S} 1$ ), and the $\mathrm{H}_{\mathrm{II}}$ phase region, which has almost the same characteristics, was frequently observed in the $1.0 \mathrm{~mm}$ diameter capillary samples.

Figure 3 shows the $\mathrm{H}_{\mathrm{II}}$ phase region in a $0.2 \mathrm{~mm}$ diameter capillary sample observed under polarized light. The structures of the sample could be distinguished without and with a crossed analyzer plate (Figure $3 a$ and $b$ ). The right side region of the sample in Figure $3 \mathrm{~b}$ was dark, except for the center along the 
(a)

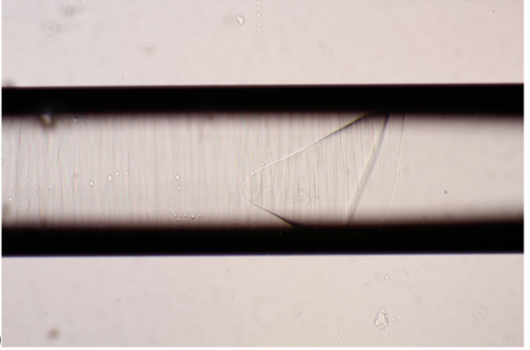

(b)

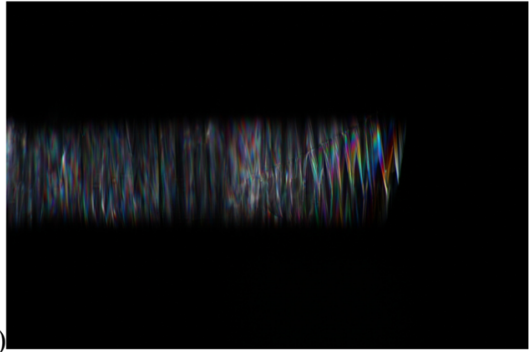

(d)
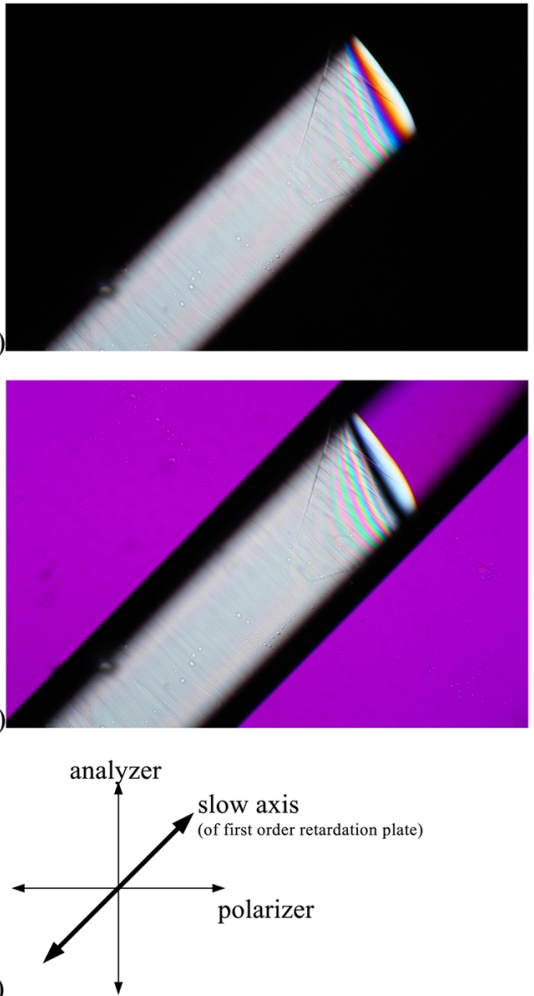

(e)

Figure 2. GME sample in a $1.0 \mathrm{~mm}$ diameter quartz capillary under polarized light. An interface region between the $\mathrm{L}_{2}$ and $\mathrm{H}_{\text {II }}$ phases is shown. The directions of polarized light from a polarizer and an analyzer when (a) parallel and $(b-d)$ perpendicular to each other. The sample was the darkest when the capillary axis was parallel to the direction of polarized light from a polarizer (b), whereas the sample was the brightest when the capillary axis was at a $45^{\circ}$ angle to the direction of polarized light from the polarizer (c). (d) Same arrangement as in (c), but with a first-order retardation plate inserted. (e) Directions of polarized light from the polarizer and analyzer. The slow axis direction of the first first-order retardation plate is also shown.

capillary axis where weak colors was observed. In contrast, the dark region in Figure $3 \mathrm{~b}$ was the brightest when the sample was rotated $45^{\circ}$ (Figure 3c). The right side region of the capillary was considered to be in the extinction position in Figure $3 \mathrm{~b}$,
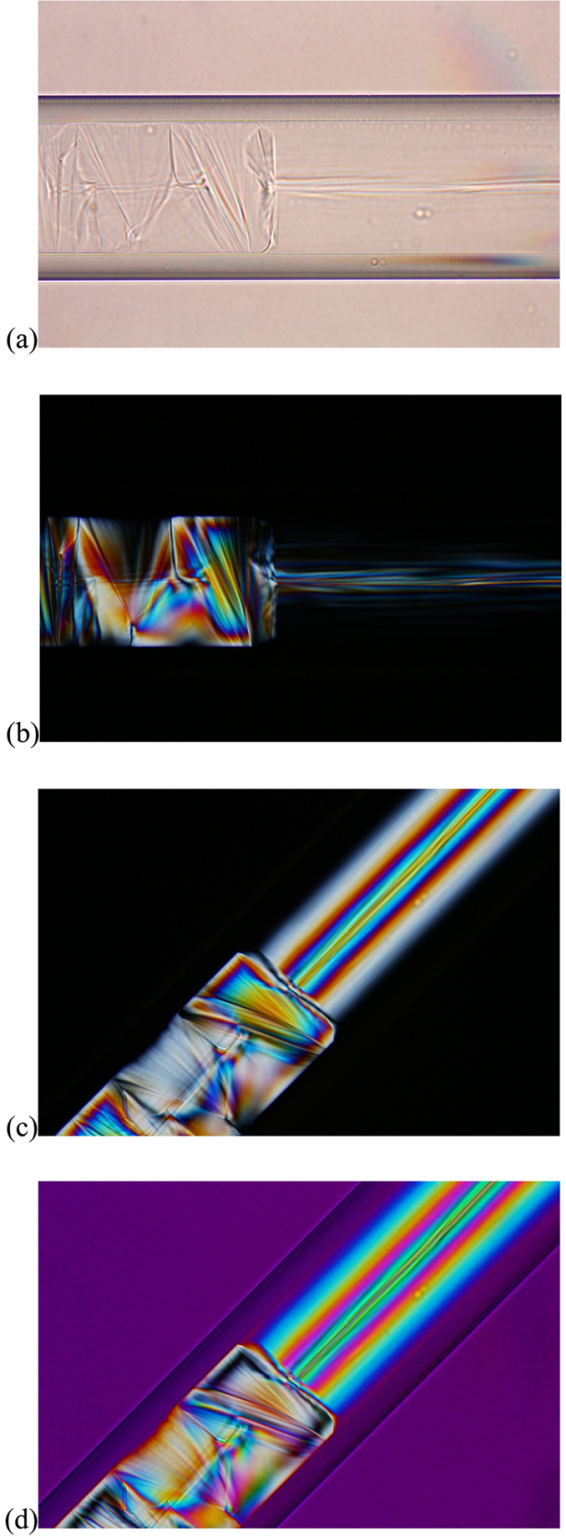

Figure 3. GME sample in a $0.2 \mathrm{~mm}$ diameter quartz capillary under polarized light. An interface region between aligned and disordered $\mathrm{H}_{\mathrm{II}}$ phases is shown. (a) Without an analyzer (a polarizer only). (b-d) Directions of polarized light from a polarizer and an analyzer when perpendicular to each other. The right side region in the capillary sample was the darkest when the capillary axis was parallel to the direction of polarized light from the polarizer (b), whereas the sample was the brightest when the capillary axis was at a $45^{\circ}$ angle to the direction of polarized light from the polarizer (c). (d) Same arrangement as in (c), but with a first order retardation plate inserted. Directions of polarized light from the polarizer and analyzer were the same as in Figure 2. The slow axis direction of the first-order retardation plate was also the same. The actual inner diameter of the capillary was $0.15 \mathrm{~mm}$.

and in the diagonal position in Figure 3c. Thus, it was concluded that the $\mathrm{H}_{\text {II }}$ phase in the right side region of the sample was aligned along the capillary axis. The left side region of the sample was a disordered $\mathrm{H}_{\mathrm{II}}$ phase but had a clear color at the capillary wall, while the colors of the right side region faded toward the wall (Figure 3c). As such, the colors of the right side region just next to the capillary wall could not be recognized. The diameter of the right side region appeared to 
be different from that of the left side region in Figure 3c, although no difference in the capillary diameter was evident (Figure 3a). This suggests that the $\mathrm{H}_{\mathrm{II}}$ phase of the right side region just next to the capillary wall lost birefringence. In Figure $3 \mathrm{~d}$, a color shift of the right side region was observed under a first-order retardation plate. Comparison of the color shift with the interference color chart indicated that the optical sign of birefringence for the right side region along the capillary axis was positive. For example, the border region between dark and yellow along the capillary wall in the right side region (Figure 3c) changed to (second-order) blue (Figure 3d). The length of the aligned $\mathrm{H}_{\mathrm{II}}$ region in Figure 3 was approximately $1 \mathrm{~mm}$ (Figure S2), although its length reached up to $5 \mathrm{~mm}$ in other samples. The aligned $\mathrm{H}_{\mathrm{II}}$ region, which had almost the same characteristics, was frequently observed in the $0.2 \mathrm{~mm}$ diameter capillary samples. The optical sign was different from that in the $1.0 \mathrm{~mm}$ diameter capillary sample, which indicates that two $c$ axes of the $\mathrm{H}_{\mathrm{II}}$ phase in both capillary samples were perpendicular to each other.

$\mathrm{X}$-ray diffraction from the aligned region in a $1.0 \mathrm{~mm}$ diameter capillary sample was measured at the BL40B2 beamline. The capillary sample was set vertically on a rotating stage with a vertically rotating axis. X-ray diffraction images were measured sequentially while the sample was rotated. The $\mathrm{X}$-ray diffraction changed with rotation and was dependent on the angle (Figure 4). Figure 4a shows the X-ray diffraction at $2^{\circ}$, and almost the same diffraction images were observed repeatedly at every $60^{\circ}$ increase (Figure S3). Figure $4 \mathrm{~b}$ shows $\mathrm{X}$-ray diffraction at $32^{\circ}$, and almost the same diffraction images were observed repeatedly at every $60^{\circ}$ increase (Figure S3). The intensity distribution in reciprocal space was calculated from the sequential X-ray diffraction images (Figure $4 \mathrm{~d}$ and e). Figure $4 \mathrm{~d}$ clearly indicates the presence of a $2 \mathrm{D}$ hexagonal lattice in the sample and the lattice plane is almost perpendicular to the capillary axis. Figure $4 a, b$, and e shows intensity distributions along the axis normal to the hexagonal lattice plane. The intensity distribution is due to fluctuations of the hexagonal lattice plane in the sample and the limited thickness of the plane along the normal axis. The axis normal to the hexagonal lattice plane was tilted approximately $3^{\circ}$ from the capillary axis. The lattice constant of the hexagonal lattice was determined to be $5.85 \mathrm{~nm}$. Fluctuation of cylinder direction was estimated around $\pm 5^{\circ}$ because full widths at half maxima of diffraction intensities along arc directions in Figure $4 a$ and $b$ were around $10^{\circ}$.

$\mathrm{X}$-ray diffraction from the aligned region in a $0.2 \mathrm{~mm}$ diameter capillary sample measured at the BL40B2 beamline was almost the same as that for a $1.0 \mathrm{~mm}$ diameter capillary sample. Diffraction images revealed a hexagonal pattern, but the pattern was unchanged during capillary rotation (data not shown). Therefore, diffraction from the sample was measured with an X-ray microbeam ( $5 \mu \mathrm{m}$ diameter) at the BL40XU beamline. Figure 5 and Figure S4 show X-ray diffraction images from the sample obtained with an X-ray microbeam. The diffraction images obtained from the left and right sides show hexagonal patterns (Figure 5a and $c$ ), where the hexagonal lattice plane in the sample was perpendicular to the X-ray microbeam. In contrast, the diffraction image from the center has different characteristics; diffraction spots on the vertical axis through the beam center were elongated along the horizontal direction and the intensities of hexagonal diffraction spots apart from the vertical axis were weak (Figure 5b). Elongated diffraction spots were also observed in the $1.0 \mathrm{~mm}$ diameter (a)

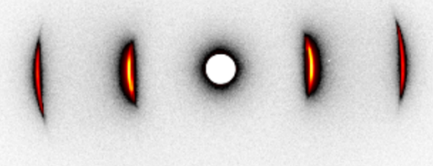

(b)
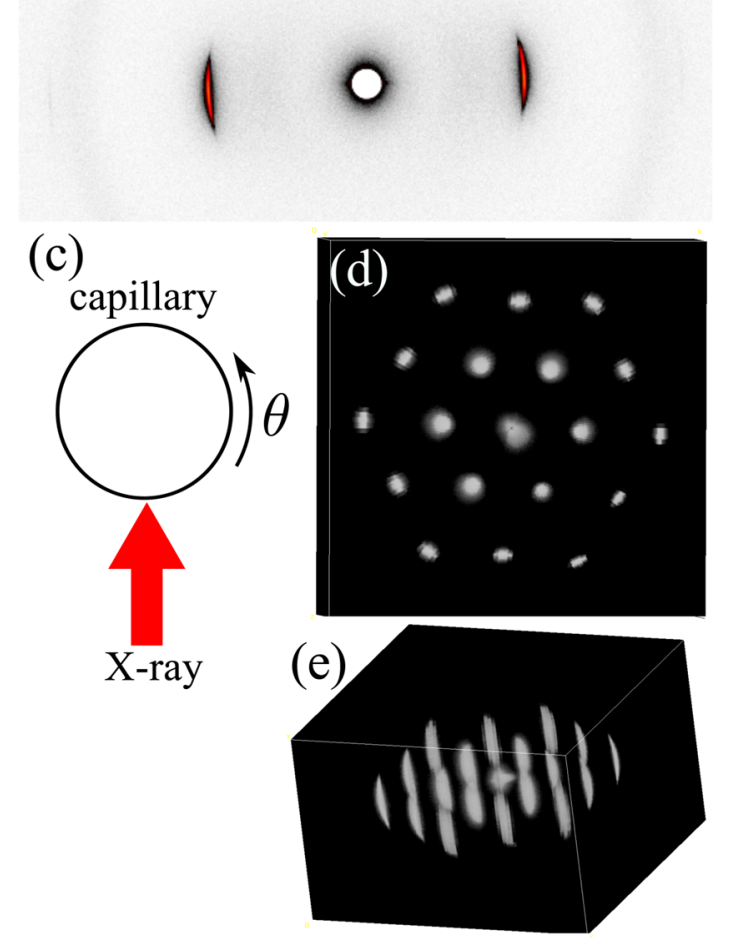

Figure 4. X-ray diffraction at (a) $2^{\circ}$ and (b) $32^{\circ}$ from the $1.0 \mathrm{~mm}$ diameter capillary sample. (c) Schematic of X-ray exposure to the capillary. The capillary sample was rotated continuously around the capillary axis from 0 to $360^{\circ}$, and X-ray images with $1 \mathrm{~s}$ exposure and $2^{\circ}$ steps were measured sequentially. Calculated intensity distribution in reciprocal space viewed almost along (d) the capillary axis and (e) from an oblique angle.

capillary sample when the X-ray beam was perpendicular to the $c$-axis of the hexagonal lattice plane (Figure $4 a$ and $b$ ). Thus, the hexagonal phase is present at the center of the $0.2 \mathrm{~mm}$ diameter capillary sample, where the $c$-axis is parallel to the horizontal direction and perpendicular to the X-ray microbeam. The same result was also observed in the same sample when rotated around the capillary axis. The lattice constant of the hexagonal lattice was $4.89 \mathrm{~nm}$.

We consider two arrangements to explain the $\mathrm{H}_{\mathrm{II}}$ orientations in the capillaries observed in these results (Figure 6). X-ray diffraction data for the $1.0 \mathrm{~mm}$ diameter capillary sample clearly showed that hexagonal cylinders of the $\mathrm{H}_{\mathrm{II}}$ phase were almost parallel to the capillary axis (Figure 4), which is supported by the diagonal position observed under polarized light (Figure 2c). However, weak colors were observed at the extinction position under polarized light (Figure $2 b$ ), although the weak colors could not be observed if the sample was oriented perfectly. Thus, the sample fluctuated in the direction of the hexagonal cylinder axis (Figure 6a). The fluctuation was estimated as around $\pm 5^{\circ}$ on average (Figure $4 \mathrm{a}$ and $\mathrm{b}$ ). X-ray diffraction data of the $0.2 \mathrm{~mm}$ diameter capillary sample 
(a)

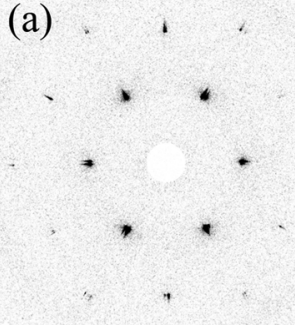

(c)

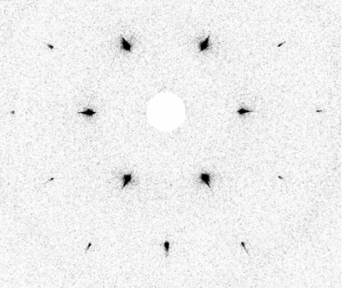

(d)

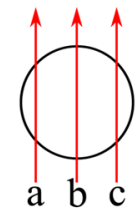

Figure 5. X-ray diffraction from the $0.2 \mathrm{~mm}$ diameter capillary sample. The diameter of the X-ray microbeam was approximately $5 \mu \mathrm{m}$. Diffractions from (a) the left side at $15 \mu \mathrm{m}$ from the left capillary wall (55 $\mu \mathrm{m}$ from the center of the capillary), (b) the center, and (c) the right side at $15 \mu \mathrm{m}$ from the right capillary wall $(55 \mu \mathrm{m}$ from the center of the capillary). The actual inner diameter of the capillary was $140 \mu \mathrm{m}$. The capillary axis of the sample was parallel to the vertical axis of the images. (d) Schematic of exposure positions in the capillary, where the circle represents a cross section of the capillary.

showed a position-dependent change in the orientation of the hexagonal cylinders. The data indicated the hexagonal cylinders were ringed along the capillary wall (Figure $6 \mathrm{~b}$ ). When X-rays pass through near the wall, the X-ray beam is almost parallel to the direction of the hexagonal cylinders (Figure 5a and $c$ ), whereas the X-rays are perpendicular to the direction of the hexagonal cylinders at the center of the capillary (Figure $5 b$ ). With this arrangement, rotation around the capillary axis does not change the orientation of the ringed cylinders. The arrangement corresponds well with the observation under polarized light, where the diagonal and extinction positions were the same as that for the $1.0 \mathrm{~mm}$ diameter capillary sample, but the optical signs were opposite. The direction of the hexagonal cylinders in the $0.2 \mathrm{~mm}$ diameter capillary was perpendicular to that in the $1.0 \mathrm{~mm}$ diameter capillary, which results in the opposite signs. A dark region was observed near the capillary wall in the $0.2 \mathrm{~mm}$ diameter capillary sample (Figure 3c). If we consider the arrangement in Figure 6b, then polarized light that passes through near the capillary wall is parallel to the hexagonal cylinders. However, there is no anisotropy of the $\mathrm{H}_{\mathrm{II}}$ phase to polarized light; therefore, the region is dark under crossed polarized illumination. Both arrangements presented in Figure 6 explain the observed data well. In each area of a capillary, only one arrangement was observed. Therefore, two types of monodomains of the $\mathrm{H}_{\text {II }}$ phase are considered to be possible with different arrangements.

\section{DISCUSSION}

We have presented two arrangements of cylinders in monodomains of the $\mathrm{H}_{\text {II }}$ phase: straight cylinders (Figure 6a) and ringed cylinders (Figure $6 \mathrm{~b}$ ). Both orientations seem to be affected by the capillary. In the straight cylinders, both edges of the cylinders, which form a hexagonal lattice plane, are exposed (a)

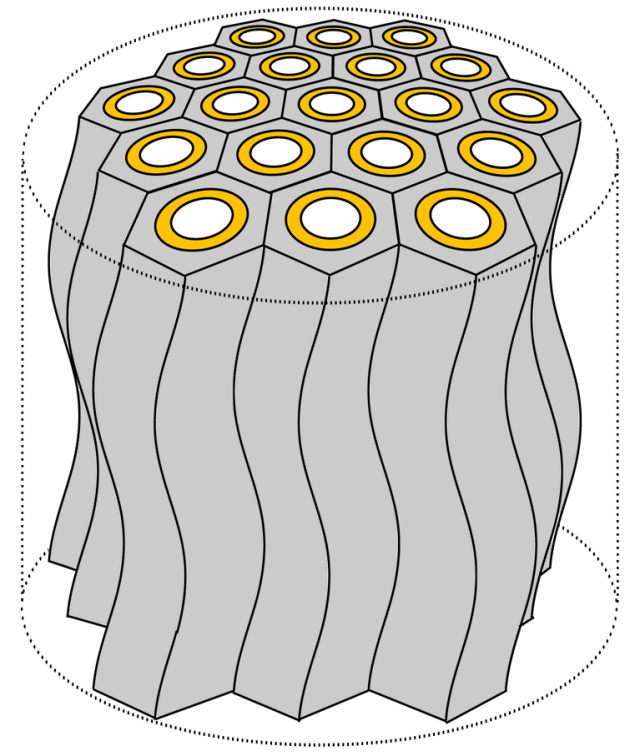

(b)

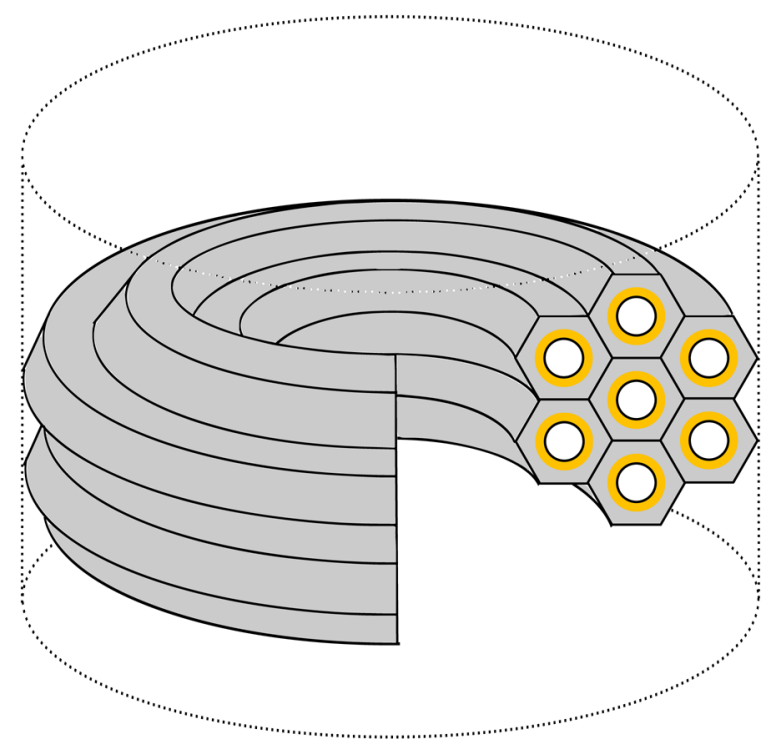

Figure 6. Two arrangements of hexagonal cylinders of the $\mathrm{H}_{\text {II }}$ phase in a capillary. (a) Straight cylinders with undulation and (b) ringed cylinders. A part of the ringed cylinders is not shown to clarify the hexagonal lattice plane in (b). The $\mathrm{H}_{\mathrm{II}}$ phase touches the capillary wall with one of the $\langle 10\rangle$ directions in a hexagonal plane facing toward the wall in (b). The cylindrical direction of the $\mathrm{H}_{\text {II }}$ phase is almost parallel to the capillary axis in (a), but the orientational relationship between one of the $\langle 10\rangle$ directions and the capillary wall in (b) is not kept in (a).

to water or the $\mathrm{L}_{2}$ phase, whereas in the ringed cylinders, there is no edge with the lattice planes because they can form closed rings. One end of a cylinder would be able to encounter the other end in a small diameter capillary during $\mathrm{H}_{\text {II }}$ phase formation, but would be unable to do so in a large diameter capillary. We showed data of straight cylinders in a $1.0 \mathrm{~mm}$ capillary and ringed cylinders in a $0.2 \mathrm{~mm}$. We also examined capillaries with diameters of $0.1,0.3$, and $0.5 \mathrm{~mm}$. The $\mathrm{H}_{\text {II }}$ phase of ringed cylinders were formed mainly in 0.1 or $0.3 \mathrm{~mm}$ 
capillaries. While straight cylinders were formed mainly in a 0.5 $\mathrm{mm}$ cylinder. But we observed small regions of straight cylinders in $0.1,0.3 \mathrm{~mm}$ capillaries and ringed cylinders in 0.5 $\mathrm{mm}$ one. If two type of cylinders were coexisted in a capillary, the $\mathrm{H}_{\text {II }}$ phase with straight cylinders was observed near the open edge of the capillary but that with ringed cylinders at far (Figure S5).

To align the $\mathrm{H}_{\mathrm{I}}$ phase, Clerc et al. used a rectangular glass capillary tube with a thickness of less than $0.2 \mathrm{~mm} .{ }^{10}$ The cylindrical direction of $\mathrm{H}_{\mathrm{I}}$ phase in the rectangular tube was parallel to the flat surface and often along the axis of the tube. Recently, Squires et al. reported on the $\mathrm{H}_{\text {II }}$-cubic phase transition using an $\mathrm{H}_{\mathrm{II}}$ phase aligned on the inner wall of a capillary. ${ }^{14}$ These alignment methods produced structures close to the straight cylinder arrangement (Figure 6a). However, there have been no reports on the production of ringed cylinders (Figure 6b). Hexosomes of straight cylinders ${ }^{24,25}$ and ringed cylinders ${ }^{26,27}$ have been reported at the nanoscale. The ringed cylinders of the $\mathrm{H}_{\text {II }}$ phase was also found as an inclusion among lamellae in rod outer segments of frog retina. ${ }^{28}$ The deformation of cylinders in a ringed shape can occur with zero elastic strain energy. ${ }^{2,29}$ Therefore, both arrangements presented in Figure 6 would be universal structures of the hexagonal phase confined to a limited size.

In the method for alignment of the $\mathrm{H}_{\mathrm{I}}$ phase, two flat and thin glass walls with a gap up to $0.2 \mathrm{~mm}$ were required. ${ }^{10,30}$ The results clearly showed the presence of the glass wall was necessary to align the $\mathrm{H}_{\mathrm{I}}$ phase. In contrast, we have used a cylindrical capillary with a $1.0 \mathrm{~mm}$ diameter round wall to align the $\mathrm{H}_{\text {II }}$ phase in a straight cylinder form. Thus, the straight cylinder of the $\mathrm{H}_{\mathrm{II}}$ phase does not align to the capillary wall but to the capillary axis direction. One fixed direction does not face the capillary wall in a monodomain of straight cylinders (Figure 6a). In the formation of straight cylinders of the $\mathrm{H}_{\text {II }}$ phase, the growth direction of the cylinder is limited to the direction of the capillary axis, i.e., the length direction. In contrast, ringed cylinders of the $\mathrm{H}_{\text {II }}$ phase in a $0.2 \mathrm{~mm}$ diameter capillary align to the wall. One of the $\{10\}$ diffraction spots was always observed at the direction perpendicular to the capillary axis (Figure 5), which indicates that one of the $\langle 10\rangle$ directions in a ringed cylinder always faces the capillary wall. Therefore, the wall is necessary to form ring cylinders of the $\mathrm{H}_{\text {II }}$ phase.

Undulation of the cylindrical direction of the $\mathrm{H}_{\mathrm{I}}$ phase has been reported. ${ }^{30,31}$ In the present work, a stripe pattern was observed in the straight cylinders of the $\mathrm{H}_{\text {II }}$ phase, which is considered to be due such an undulation (Figures $2 a$ and $b$ ). The pitch of the stripe pattern was less than $50 \mu \mathrm{m}$ at the region near the interface between the $\mathrm{L}_{2}$ and $\mathrm{H}_{\mathrm{II}}$ phases. The pitch seemed to be shorter at a distance far from the region; however, the thickness of the sample prevented an exact estimation of the pitch. The undulation in the $\mathrm{H}_{\mathrm{I}}$ phase was considered to be due to a thermomechanical undulation of the columns. ${ }^{30}$ The same theory may be applicable to that in the $\mathrm{H}_{\text {II }}$ phase.

The lattice constant of the monodomain with straight cylinders was $5.85 \mathrm{~nm}$, while that with ringed cylinders was 4.89 $\mathrm{nm}$. Almost the same values were observed in other samples. The maximum value for the lattice constant of the $\mathrm{H}_{\text {II }}$ phase was around $5.8 \mathrm{~nm}$ in the capillary samples observed here; therefore, the monodomain in the $1.0 \mathrm{~mm}$ diameter capillary was considered to be fully hydrated. Barauskas et al. showed that the lattice constant of the $\mathrm{H}_{\text {II }}$ phase of GME increased from 4.8 to $5.8 \mathrm{~nm}$ as the water content increased from 13 to
$27 \%$ at $25^{\circ} \mathrm{C} .{ }^{22}$ The lattice constant remained unchanged upon further addition of water due to phase separation between the $\mathrm{H}_{\text {II }}$ phase and water. Thus, the water content of the $\mathrm{H}_{\text {II }}$ monodomain with straight cylinders is considered to be around $27 \%$, and that with the ringed cylinders around $13 \%$. In Figure $2 \mathrm{a}$, a stripe pattern was observed in the $\mathrm{H}_{\mathrm{II}}$ region. If the region were occupied fully with the $\mathrm{H}_{\text {II }}$ phase, then the stripes would not be observed because the refraction index in the region would be homogeneous. However, the $\mathrm{H}_{\mathrm{II}}$ phase was separated from water in this region; therefore, the difference in the refractive indexes between the $\mathrm{H}_{\mathrm{II}}$ monodomain with undulated straight cylinders and water would result in the stripe pattern. The monodomain with ringed cylinders would be stabilized with a lower water content and by interaction with the capillary wall; therefore, the growth rate of the $\mathrm{H}_{\text {II }}$ phase region in the $0.2 \mathrm{~mm}$ diameter capillary was slower than that in the $1.0 \mathrm{~mm}$ diameter capillary. Figures S1 and S2 show both capillaries soaked in water for 3 weeks, where the length of the $\mathrm{H}_{\text {II }}$ phase in the $0.2 \mathrm{~mm}$ diameter capillary was shorter than that in the $1.0 \mathrm{~mm}$ diameter capillary. The monodomain with ringed cylinders, of which the water content was low, would retard water inflow into the capillary. In addition, the monodomain with ringed cylinders has a fault in structure at the center (Figure 6b), as indicated by the weak colored region at the center of the right side half of the capillary in Figure $3 b$, and the same region had a different refraction index from that of the $\mathrm{H}_{\mathrm{II}}$ phase (Figure 3a). This may indicate there is a high water content at the fault of the monodomain with ringed cylinders.

We previously reported a method for the preparation of a single crystal region of the cubic II phase. ${ }^{15}$ In the method, slow out diffusion of the organic solvent from the capillary enabled the slow phase transition from the $\mathrm{L}_{3}$ to the cubic II phase along the capillary axis and slow growth of a single crystal region. A similar method was employed here to prepare the two distinct monodomains of the $\mathrm{H}_{\text {II }}$ phase with straight cylinders and ringed cylinders. A slow phase transition from the $\mathrm{L}_{2}$ to $\mathrm{H}_{\mathrm{II}}$ phase in a capillary is important to prepare both monodomains of the $\mathrm{H}_{\text {II }}$ phase. In the formation of a monodomain with ringed cylinders, a slow phase transition appears to be necessary because a rapid increase in water would not allow formation of this monodomain, of which the water content is almost at the lowest level observed for the $\mathrm{H}_{\mathrm{II}}$ phase. ${ }^{22}$

The alignment method described here could be applied to several research areas. The $\mathrm{L}_{2}-\mathrm{H}_{\mathrm{II}}$ phase transition of GME was employed in this work. Both phases are commonly observed in amphiphile/water systems; therefore, this method could be applied to other materials for the alignment of $\mathrm{H}_{\mathrm{II}}$ phases. Phase transitions from or to the $\mathrm{H}_{\text {II }}$ phase are one of the candidate to be applied the alignment method. ${ }^{32,33}$ Recently, Squires et al. reported a $\mathrm{H}_{\mathrm{II}}$-cubic phase transition, in which the $\mathrm{H}_{\text {II }}$ phase was aligned along a capillary wall in a different manner from the method presented in this work. ${ }^{14}$ Their method can produce the $\mathrm{H}_{\text {II }}$ phase aligned on the inner wall of a capillary, where the central region of the capillary is occupied by water. In contrast, aligned straight cylinders of $\mathrm{H}_{\mathrm{II}}$ phase prepared with the present method do not align to the capillary wall but instead occupy the entire inner region of the capillary. Thus, samples prepared using the present method would be suitable for study with rotating crystal methods, ${ }^{17,18}$ which could reveal new information on the phase transition. Epitaxial relationships of the $\mathrm{La}-\mathrm{H}_{\mathrm{II}}$ phase transition has been proposed based on X-ray diffraction studies with polycrystal samples. ${ }^{34,35}$ The alignment method of the $\mathrm{H}_{\mathrm{II}}$ phase in this 
paper might contribute to researches of the epitaxial relationships. The $\mathrm{H}_{\text {II }}$ phase of lyotropic liquid crystals are also used as templates ${ }^{36}$ for the production of nanostructured materials. The two distinct arrangements of hexagonal cylinders of the $\mathrm{H}_{\mathrm{II}}$ phase have characteristic configurations, long straight cylinders and unique ringed cylinders, which could find effective application as templates.

\section{CONCLUSION}

A simple method was developed to prepare two types of $\mathrm{H}_{\mathrm{II}}$ phase monodomains of GME in capillaries. The first monodomain of the $\mathrm{H}_{\mathrm{II}}$ phase has straight cylinders where the cylindrical axes are almost parallel to the capillary axis, although the cylinders undulate slightly. The lattice constant of the monodomain was $5.85 \mathrm{~nm}$, which is comparable to that of the fully hydrated $\mathrm{H}_{\text {II }}$ phase. The second monodomain has ringed cylinders, where one of the $\langle 10\rangle$ directions in the hexagonal plane is always perpendicular to the capillary wall, and a ring-like structure is formed around the wall of the capillary. The lattice constant of the monodomain was $4.89 \mathrm{~nm}$ and the water content was approximately $13 \%$, which indicates the monodomain was not fully hydrated. The capillary wall and the low water content are considered to stabilize the monodomains with ringed cylinders. The method presented here for the preparation of both monodomains is expected to contribute to further research on the $\mathrm{H}_{\text {II }}$ phase in fundamental science and in practical applications.

\section{ASSOCIATED CONTENT}

\section{S Supporting Information}

The Supporting Information is available free of charge on the ACS Publications website at DOI: 10.1021/acs.langmuir.6b00996.

Full views of capillaries for Figures 2 and 3; sequential Xray diffraction images for Figures 4 and 5 (PDF)

\section{AUTHOR INFORMATION}

\section{Corresponding Author}

*E-mail: oka.toshihiko@shizuoka.ac.jp.

\section{Notes}

The authors declare no competing financial interest.

\section{ACKNOWLEDGMENTS}

NIKOL Selachyl alcohol was kindly gifted by Nikko Chemicals Co. Ltd.. This work was supported by JSPS KAKENHI Grant Number 15K05243 to T.O. Part of this research is based on a Cooperative Research Project of the Research Institute of Electronics, Shizuoka University. The synchrotron radiation experiments were performed using BL40B2 and BL40XU at SPring-8 with the approval of the Japan Synchrotron Radiation Research Institute (JASRI) (Proposal No. 2015A1295, 2015B1530, 2015B1351). Preliminary SAXS experiments were done at Molecular Structure Analysis Section of Shizuoka University RIGST.

\section{REFERENCES}

(1) Hyde, S. T. Identification of Lyotropic Liquid Crystalline Mesophases. In Handbook of Applied Surface and Colloid Chemistry; J. Wiley \& Sons: Chichester, 2002; Vol. 16, pp 299-332.

(2) Seddon, J. M. Structure of the Inverted Hexagonal (HII) Phase, and Non-Lamellar Phase Transitions of Lipids. Biochim. Biophys. Acta, Rev. Biomembr. 1990, 1031 (1), 1-69.
(3) Israelachvili, J. N. Intermolecular and Surface Forces, revised third ed.; Academic Press: New York, 2011.

(4) Kresge, C. T.; Leonowicz, M. E.; Roth, W. J.; Vartuli, J. C.; Beck, J. S. Ordered Mesoporous Molecular Sieves Synthesized by a LiquidCrystal Template Mechanism. Nature 1992, 359 (6397), 710-712.

(5) Tanev, P. T.; Pinnavaia, T. J. A Neutral Templating Route to Mesoporous Molecular Sieves. Science 1995, 267 (5199), 865-867.

(6) Huang, L. m.; Wang, H. t.; Wang, Z. b.; Mitra, A.; Bozhilov, K. n.; Yan, Y. s. Nanowire Arrays Electrodeposited from Liquid Crystalline Phases. Adv. Mater. 2002, 14 (1), 61-64.

(7) Drummond, C. J.; Fong, C. Surfactant Self-Assembly Objects as Novel Drug Delivery Vehicles. Curr. Opin. Colloid Interface Sci. 1999, 4 (6), 449-456.

(8) Uchino, T.; Murata, A.; Miyazaki, Y.; Oka, T.; Kagawa, Y. Glyceryl Monooleyl Ether-Based Liquid Crystalline Nanoparticles as a Transdermal Delivery System of Flurbiprofen: Characterization and in Vitro Transport. Chem. Pharm. Bull. 2015, 63 (5), 334-340.

(9) Rancon, Y.; Charvolin, J. Epitaxial Relationships during Phase Transformations in a Lyotropic Liquid Crystal. J. Phys. Chem. 1988, 92 (9), 2646-2651.

(10) Clerc, M.; Levelut, A. M.; Sadoc, J. F. Transitions between Mesophases Involving Cubic Phases in the Surfactant-Water Systems. Epitaxial Relations and Their Consequences in a Geometrical Framework. J. Phys. II 1991, 1 (10), 1263-1276.

(11) Ramos, L.; Molino, F.; Porte, G. Shear Melting in Lyotropic Hexagonal Phases. Langmuir 2000, 16 (14), 5846-5848.

(12) Ramos, L. Time-Resolved Synchrotron X-Ray Scattering of the Crystallization of a Soft Hexagonal Columnar Crystal. Langmuir 2004, 20 (6), 2215-2219.

(13) Pan, J.; Heberle, F. A.; Petruzielo, R. S.; Katsaras, J. Using SmallAngle Neutron Scattering to Detect Nanoscopic Lipid Domains. Chem. Phys. Lipids 2013, 170-171, 19-32.

(14) Squires, A. M.; Akbar, S.; Tousley, M. E.; Rokhlenko, Y.; Singer, J. P.; Osuji, C. O. Experimental Evidence for Proposed Transformation Pathway from the Inverse Hexagonal to Inverse Diamond Cubic Phase from Oriented Lipid Samples. Langmuir 2015, 31 (28), 7707-7711.

(15) Oka, T.; Hojo, H. Single Crystallization of an Inverse Bicontinuous Cubic Phase of a Lipid. Langmuir 2014, 30 (28), $8253-8257$.

(16) Engström, S.; Wadsten-Hindrichsen, P.; Hernius, B. Cubic, Sponge, and Lamellar Phases in the Glyceryl Monooleyl EtherPropylene Glycol-Water System. Langmuir 2007, 23 (20), 1002010025.

(17) Oka, T. Transformation between Inverse Bicontinuous Cubic Phases of a Lipid from Diamond to Primitive. Langmuir 2015, 31 (10), $3180-3185$.

(18) Oka, T. Transformation between Inverse Bicontinuous Cubic Phases of a Lipid from Diamond to Gyroid. Langmuir 2015, 31 (41), 11353-11359.

(19) Schneider, C. A.; Rasband, W. S.; Eliceiri, K. W. NIH Image to ImageJ: 25 Years of Image Analysis. Nat. Methods 2012, 9 (7), 671675 .

(20) Hara, T.; Tanaka, T.; Seike, T.; Bizen, T.; Maréchal, X.; Kohda, T.; Inoue, K.; Oka, T.; Suzuki, T.; Yagi, N.; et al. In-Vacuum X-Ray Helical Undulator for High Flux Beamline at SPring-8. Nucl. Instrum. Methods Phys. Res., Sect. A 2001, 467-468, 165-168.

(21) Ohta, N.; Oka, T.; Inoue, K.; Yagi, N.; Kato, S.; Hatta, I. Structural Analysis of Cell Membrane Complex of a Hair Fibre by Micro-Beam X-Ray Diffraction. J. Appl. Crystallogr. 2005, 38 (2), 274279.

(22) Barauskas, J.; Švedaitè, I.; Butkus, E.; Razumas, V.; Larsson, K.; Tiberg, F. Synthesis and Aqueous Phase Behavior of 1-Glyceryl Monooleyl Ether. Colloids Surf., B 2005, 41 (1), 49-53.

(23) Awaya, H. Introduction of polarized light microscope for polymer materials (in Japanese); Agne Gijutsu Center: Tokyo, 2001.

(24) Boyd, B. J.; Whittaker, D. V.; Khoo, S.-M.; Davey, G. Hexosomes Formed from Glycerate surfactants-Formulation as a Colloidal Carrier for Irinotecan. Int. J. Pharm. 2006, 318 (1-2), 154162. 
(25) Boyd, B. J.; Rizwan, S. B.; Dong, Y.-D.; Hook, S.; Rades, T. SelfAssembled Geometric Liquid-Crystalline Nanoparticles Imaged in Three Dimensions: Hexosomes Are Not Necessarily Flat Hexagonal Prisms. Langmuir 2007, 23 (25), 12461-12464.

(26) de Campo, L.; Yaghmur, A.; Sagalowicz, L.; Leser, M. E.; Watzke, H.; Glatter, O. Reversible Phase Transitions in Emulsified Nanostructured Lipid Systems. Langmuir 2004, 20 (13), 5254-5261.

(27) Sagalowicz, L.; Michel, M.; Adrian, M.; Frossard, P.; Rouvet, M.; Watzke, H. J.; Yaghmur, A.; De Campo, L.; Glatter, O.; Leser, M. E. Crystallography of Dispersed Liquid Crystalline Phases Studied by Cryo-Transmission Electron Microscopy. J. Microsc. 2006, 221 (2), 110-121.

(28) Corless, J. M.; Joseph Costello, M. Paracrystalline Inclusions Associated with the Disk Membranes of Frog Retinal Rod Outer Segments. Exp. Eye Res. 1981, 32 (2), 217-228.

(29) Kleman, M. Developable Domains in Hexagonal Liquid Crystals. J. Phys. (Paris) 1980, 41 (7), 737-745.

(30) Oswald, P.; Geminard, J. C.; Lejcek, L.; Sallen, L. Nonlinear Analysis of Stripe Textures in Hexagonal Lyotropic Mesophases. J. Phys. II 1996, 6 (2), 281-303.

(31) Impéror-Clerc, M.; Davidson, P. An X-Ray Scattering Study of Flow-Aligned Samples of a Lyotropic Liquid-Crystalline Hexagonal Phase. Eur. Phys. J. B 1999, 9 (1), 93-104.

(32) Oka, T.; Tsuboi, T.; Saiki, T.; Takahashi, T.; Alam, J. M.; Yamazaki, M. Initial Step of pH-Jump-Induced Lamellar to Bicontinuous Cubic Phase Transition in Dioleoylphosphatidylserine/ Monoolein. Langmuir 2014, 30 (27), 8131-8140.

(33) Oka, T.; Saiki, T.; Alam, J. M.; Yamazaki, M. Activation Energy of the Low-pH-Induced Lamellar to Bicontinuous Cubic Phase Transition in Dioleoylphosphatidylserine/Monoolein. Langmuir 2016, 32 (5), 1327-1337.

(34) Rappolt, M.; Hickel, A.; Bringezu, F.; Lohner, K. Mechanism of the Lamellar/Inverse Hexagonal Phase Transition Examined by High Resolution X-Ray Diffraction. Biophys. J. 2003, 84 (5), 3111-3122.

(35) Yaghmur, A.; Sartori, B.; Rappolt, M. Self-Assembled Nanostructures of Fully Hydrated Monoelaidin-Elaidic Acid and Monoelaidin-Oleic Acid Systems. Langmuir 2012, 28 (26), 1010510119.

(36) He, W.; Fu, Y.; Andersson, M. Morphological Control of Calcium Phosphate Nanostructures Using Lyotropic Liquid Crystals. J. Mater. Chem. B 2014, 2 (21), 3214-3220. 